\title{
Survivor rates of new entrants into an undergraduate degree programme under clearance uncertainty
}

\author{
Virtue U. Ekhosuehi ${ }^{1, *}$, and Francis O. Oyegue ${ }^{1}$ \\ ${ }^{1}$ Department of Mathematics, University of Benin, P.M.B. 1154, Benin City, Nigeria
}

Received 2016/08/23; Accepted 2016/09/19; Published Online 2016/11/1

Copyright (C) 2016, African Journal of Applied Statistics (AJAS) and Probability African Society (SPAS). All rights reserved

\begin{abstract}
Considering the clearance exercise in a Nigerian university setting, this study attempts to answer the question of the following sort: 'What is the survivor rate of candidates offered (provisional) admission into a course of study in the face of clearance uncertainty?' We represent the problem as a system of differential equations within the framework of a network model with constant risk and then solve it using the Laplace transform. Rather than relying on steady-states fractional flow rates, we provide a transient solution to the problem. The survivor function for each entry level derived for the problem sheds some light on situations where a decision is to be made on two (or more) preferred courses and thus evades possible trauma in the application process.
\end{abstract}

\section{Résumé :}

Cette étude prend place dans la problématique de l'admission des étudiants dans les universités nigériannes. Elle se propose de répondre aux questions semblables à celle-ci : quel est le taux de survie (final) des candidats provisoirement admis à un programme dans une situation d'admission finale incertaine? Nous modélisons ce problème grâce à un système d'équations différentielles dans le cadre d'un modèle de réseau à risque constant. La transformation de Laplace est notre outil pour résoudre le problème théorique.

Key words: Admission, clearance, Laplace transform, survivor function, transient solution. AMS 2010 Mathematics Subject Classification : 97K80; 97M40.

\section{Presented by Dr Serigne Lo, University of Sydney, Australia, Member of the Corresponding Editors Board}

\footnotetext{
${ }^{*}$ Corresponding author Virtue U. Ekhosuehi: virtue.ekhosuehi@uniben.edu

Francis O. Oyegue : fooyegue@yahoo.com
} 
Ekhosuehi V.U. and Oyegue, F.O. African Journal of Applied Statictics, Vol. 3 (1), 2016, 59 - 67. Survivor rates of new entrants into an undergraduate degree programme under clearance uncertainty.

\section{Introduction}

Starting with the work of [Gani (1963)] mathematical models have been used as an important tool to shed light on the educational process. The art of applying mathematical techniques to a wide variety of problems in education has gained prominence in the literature (for instance, [Jimenez and Salas-Velasco (2000)]; [Mancebon and Muniz (2008)]; [Nicholls (2009)];[Ekhosuehi and Osagiede (2013)];[Johnes (2015)]; [Ekhosuehi (2016)]). These studies have shown that research in education is a worthwhile venture to educational planners, managers and consumers of education. Our present study is centred on the admission stage of the educational process in the context of the university setting in Nigeria. However, the survivor function derived in this paper may be extended to other degree awarding institutions in Nigeria such as: Nigerian Defence Academy, Nigerian Police Academy, and some colleges of: education, technology, agriculture, etc. Without loss of generality, we use the term university and institution interchangeably. More specifically, we consider the clearance of a cohort of candidates who are offered (provisional) admission into a course of study in the university. A cohort of candidates admitted in the same session is usually identified by their matriculation (or registration) number. For example, in the University of Benin with which the authors are acquainted, the first two digits of the matriculation number indicate the year of entry.

Candidates are normally admitted into either the first year (100 Level) or the second year (200 Level) of an undergraduate degree programme in the university. For a candidate to be considered for admission into the first year of any course of study (course for short) in a university full-time programme, the candidate must sit for the Unified Tertiary Matriculation Examination (UTME) and obtain a reasonable score. The UTME is conducted by the Joint Admission and Matriculation Board (JAMB). Similarly, candidates for direct entry admission must apply through JAMB to the university. The university has the prerogative to admit candidates into a course based on a certain cut-off mark (i.e., merit) and other approved criteria (such as catchment area, educationally less developed area and other selection criteria applicable to the institution) in consultation with JAMB, provided the capacity requirement for the course is not exceeded. The capacity requirement refers to the maximum number of students that will not cause undue strain on facilities in the university.

The practice of admission in most Nigerian universities is that it is done in batches. The release of a list of candidates for (provisional) admission is usually followed by a clearance exercise. The clearance exercise is carried out in the first year of entry to determine the eligibility of candidates for the course for which provisional admission has been offered. During this exercise, the candidate may be accepted as a student into the course of study or rejected. The rejection of a candidate may be due to the failure of the candidate to present the required documents for clearance (e.g., those awaiting results), deficiency in the minimum entry requirement into the course of study, non-payment of a non-refundable acceptance fee, voluntary withdrawal, etc. A candidate may also be relocated to another course if the candidate does not meet the admission requirements for the course that admission was initially offered, but satisfies that of the latter. The relocation of a candidate to another course is treated here as a rejection from the course that admission was initially offered. The survivor function derived in this study is centred on the course that admission

Journal home page: www.jafristatap.net 
Ekhosuehi V.U. and Oyegue, F.O. African Journal of Applied Statictics, Vol. 3 (1), 2016, 59 - 67. Survivor rates of new entrants into an undergraduate degree programme under clearance uncertainty.

was initially offered.

Considering the challenges candidates offered (provisional) admission into universities in Nigeria encounter, one common feature is the notion of uncertainty on whether they would be accepted or rejected. This arises from the uncertainty of the clearance exercise. This study is apt in the sense that it attempts to offer a theoretical explanation on the survivor rates of candidates who undergo the clearance exercise. We derive a system of differential equations from a network of transitions between any two states of the system, viz. the external environment (state 0), 100 Level (state 1) and 200 Level (state 2). This study contributes to the existing literature by providing a useful framework to assist candidates on the choice of courses as this would enable them evade possible trauma in the application process.

\section{Theoretical framework}

Consider a cohort of candidates of size $N(0)$ that are offered (provisional) admission into a course of study in the university (system hereafter) at the same session who should go through a clearance exercise. The candidates may enter the system at either 100 Level (state 1) or 200 Level (state 2). Let $N_{i}(t)$ be the number of candidates in State $i$ at moment $t, i=0,1,2$ and $t$ is an instant of time (in days) of a session. The network $(N, A)$ for the system with $N=\{0,1,2\}$ and $A=\{(0,1),(1,0),(0,2),(2,0)\}$ is described by the transition diagram in Figure 1.

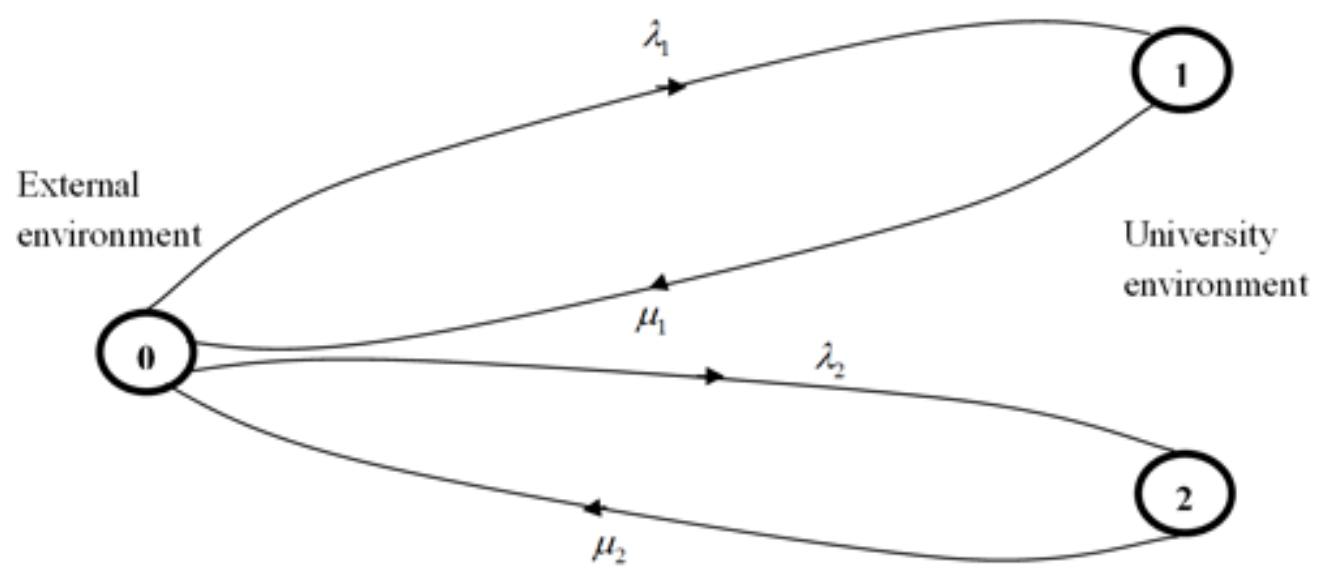

Fig. 1. Transition diagram for the admission process

The three circles represent the states that a candidate may be and the arrows indicate the changes of states which can occur. The symbol $\lambda_{i}$ is the propensity of candidates to be 
Ekhosuehi V.U. and Oyegue, F.O. African Journal of Applied Statictics, Vol. 3 (1), 2016, 59 - 67. Survivor rates of new entrants into an undergraduate degree programme under clearance uncertainty.

cleared into state $i$ and $\mu_{i}$ is the propensity of candidates to leave state $i$ in the year of entry for whatever reasons. The constancy of $\lambda_{i}$ and $\mu_{i}$ implies that the transition propensities do not depend on the time instance of the session. This would mean, for example, that the time a candidate presents himself/herself for clearance during the period of the exercise would be of no value in predicting the outcome of the exercise. The propensities $\lambda_{i}$ and $\mu_{i}$ are computed as $\lambda_{i}=\frac{\eta_{i}}{C_{i}}$ and $\mu_{i}=\frac{\vartheta_{i}}{C_{i}}$, where $\eta_{i}$ is the number of candidates cleared into state $i$ over time, $\vartheta_{i}$ is the number of candidates who leave state $i$ in the year of entry over time, and $C_{i}$ is the number of candidates offered (provisional) admission into state $i$ over time.

The proportion of candidates who remain in the system (i.e., the survivor function), $G(t)$, is given as

$$
G(t)=G_{1}(t)+G_{2}(t)
$$

where $G_{1}(t)=\frac{N_{1}(t)}{N(0)}$ is the survivor function for state $1, G_{2}(t)=\frac{N_{2}(t)}{N(0)}$ is the survivor function for state 2 , and $0 \leq G(t) \leq 1$. The relation $0 \leq G(t) \leq 1$ is used as a proxy for the uncertainty driven by the clearance exercise. If $G(t)=1$, then it is certain that everyone offered provisional admission would be eventually cleared, otherwise $0 \leq G(t)<1$.

The transition diagram in Figure 1 leads us to formulate the following differential equations for the system

$$
\begin{gathered}
\frac{d N_{0}(t)}{d t}=-\left(\lambda_{1}+\lambda_{2}\right) N_{0}(t)+\mu_{1} N_{1}(t)+\mu_{2} N_{2}(t) \\
\frac{d N_{1}(t)}{d t}=\lambda_{1} N_{0}(t)-\mu_{1} N_{1}(t) \\
\frac{d N_{2}(t)}{d t}=\lambda_{2} N_{0}(t)=\mu_{2} N_{2}(t)
\end{gathered}
$$

Since every candidate offered (provisional) admission into the system must come from a state external to the university, $N_{0}(0)=N(0), N_{1}(0)=0$ and $N_{2}(0)=0$. Rather than developing a discrete-time model for the system, we formulate the problem in continuous-time. This is because admission is done in batches and the clearance to ascertain the eligibility of candidates for a course is carried out continuously throughout the session.

We represent equations 2, 3 and 4 in a matrix-vector form as

$$
\frac{d \mathbf{N}(t)}{d t}=\Gamma \mathbf{N}(t)
$$


Ekhosuehi V.U. and Oyegue, F.O. African Journal of Applied Statictics, Vol. 3 (1), 2016, 59 - 67. Survivor rates of new entrants into an undergraduate degree programme under clearance uncertainty.

where

$$
\begin{gathered}
\frac{d \mathbf{N}(t)}{d t}=\left[\begin{array}{c}
\frac{d N_{0}(t)}{d t} \\
\frac{d N_{1}(t)}{d t} \\
\frac{d N_{2}(t)}{d t}
\end{array}\right], \\
\Gamma=\left[\begin{array}{ccc}
\left(\lambda_{1}+\lambda_{2}\right) & \mu_{1} & \mu_{2} \\
\lambda_{1} & -\mu & 0 \\
\lambda_{2} & 0 & -\mu_{2}
\end{array}\right]
\end{gathered}
$$

and

$$
\mathbf{N}(t)=\left[\begin{array}{c}
N_{0}(t) \\
N_{1}(t) \\
N_{2}(t)
\end{array}\right]
$$

so that the initial structure of the system is

$$
\mathbf{N}(0)=\left[\begin{array}{c}
N(0) \\
0 \\
0
\end{array}\right] .
$$

Let $\mathbf{M}(s)$ be the Laplace transform of $\mathbf{N}(t)$. That is

$$
\mathbf{M}(s)=\int_{0}^{\infty} \exp (-s t) \mathbf{N}(\mathbf{t}) d t
$$

By integration by parts, equation 6 simplifies to

$$
\mathbf{M}(\mathbf{s})=[s \mathbf{I}-\Gamma]^{-1} \mathbf{N}(0)
$$

where the reciprocal matrix $[s \mathbf{I}-\Gamma]^{-1}$ is given as

$$
\begin{aligned}
& {[s \mathbf{I}-\Gamma]^{-1}=\frac{1}{s(s-\alpha)(s-\beta)} \times} \\
& {\left[\begin{array}{ccc}
\left(s+\mu_{1}\right)\left(s+\mu_{2}\right) & \mu_{1}\left(s+\mu_{2}\right) & \mu_{2}\left(s+\mu_{1}\right) \\
\lambda_{1}\left(s+\mu_{2}\right) & \left(s+\lambda_{1}+\lambda_{2}\right)\left(s+\mu_{2}\right)-\mu_{2} \lambda_{2} & \mu_{2} \lambda_{1} \\
\lambda_{2}\left(s+\mu_{1}\right) & \mu_{1} \lambda_{2} & \left(s+\lambda_{1}+\lambda_{2}\right)\left(s+\mu_{1}\right)-\mu_{1} \lambda_{1}
\end{array}\right],}
\end{aligned}
$$

with 
Ekhosuehi V.U. and Oyegue, F.O. African Journal of Applied Statictics, Vol. 3 (1), 2016, 59 - 67. Survivor rates of new entrants into an undergraduate degree programme under clearance uncertainty.

$$
\begin{aligned}
\alpha & =-\frac{1}{2} \times \\
& \left(\lambda_{1}+\lambda_{2}+\mu_{1}+\mu_{2}\right. \\
& \left.+\sqrt{\left(\lambda_{1}+\lambda_{2}+\mu_{1}+\mu_{2}\right)^{2}-4\left(\lambda_{1} \mu_{2}+\lambda_{2} \mu_{1}+\mu_{1} \mu_{2}\right)}\right)<0
\end{aligned}
$$

and

$$
\begin{aligned}
& \beta=-\frac{1}{2} \times \\
& \left(\lambda_{1}+\lambda_{2}+\mu_{1}+\mu_{2}\right. \\
& \left.-\sqrt{\left(\lambda_{1}+\lambda_{2}+\mu_{1}+\mu_{2}\right)^{2}-4\left(\lambda_{1} \mu_{2}+\lambda_{2} \mu_{1}+\mu_{1} \mu_{2}\right)}\right)<0 .
\end{aligned}
$$

Resolving this reciprocal matrix into partial fractions, we get

$$
[s \mathbf{I}-\mathbf{Q}]^{-1}=\frac{1}{s} \mathbf{A}+\frac{1}{(s-\alpha)} \mathbf{B}+\frac{1}{(s-\beta)} \mathbf{C}
$$

where

$$
\mathbf{A}=\frac{1}{\alpha \beta}\left[\begin{array}{ccc}
\mu_{1} \mu_{2} & \mu_{1} \mu_{2} & \mu_{2} \mu_{1} \\
\lambda_{1} \mu_{2} & \lambda_{1} \mu_{2} & \mu_{2} \lambda_{1} \\
\lambda_{2} \mu_{1} & \mu_{1} \lambda_{2} & \lambda_{2} \mu_{1}
\end{array}\right]
$$

and

$$
\begin{gathered}
\mathbf{B}=\frac{1}{\alpha(\alpha-\beta)} \times \\
{\left[\begin{array}{cc}
\left(\alpha+\mu_{1}\right)\left(\alpha+\mu_{2}\right) \\
\lambda_{1}\left(\alpha+\mu_{2}\right) \\
\lambda_{2}\left(\alpha+\mu_{1}\right)
\end{array}\right.} \\
\begin{array}{ccc}
\mu_{1}\left(\alpha+\mu_{2}\right) \\
\left(\alpha+\lambda_{1}+\lambda_{2}\right)\left(\alpha+\mu_{2}\right)-\mu_{2} \lambda_{2}
\end{array} \\
\mu_{1} \lambda_{2}
\end{gathered}
$$

and

$$
\begin{aligned}
& \mathbf{C}=\frac{1}{\beta(\beta-\alpha)} \times
\end{aligned}
$$

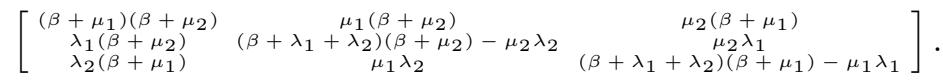

Equation 8 is equivalent to writing 
Ekhosuehi V.U. and Oyegue, F.O. African Journal of Applied Statictics, Vol. 3 (1), 2016, 59 - 67. Survivor rates of new entrants into an undergraduate degree programme under clearance uncertainty.

$$
\begin{aligned}
{[s \mathbf{I}-\Gamma]^{-1} } & =\int_{0}^{\infty} \exp (-s t) \mathbf{A} d t \\
& +\int_{0}^{\infty} \exp (-(s-\alpha) t) \mathbf{B} d t \\
& +\int_{0}^{\infty} \exp (-(s-\beta) t) \mathbf{C} d t
\end{aligned}
$$

Therefore

$$
\begin{aligned}
\mathbf{M}(s) & =[s \mathbf{I}-\Gamma]^{-1} \mathbf{N}(0) \\
& =\int_{0}^{\infty} \exp (-s t)[\mathbf{A}+\exp (\alpha t) \mathbf{B}+\exp (\beta t) \mathbf{C}] \mathbf{N}(0) d t
\end{aligned}
$$

Hence we have

$$
\mathbf{N}(t)=[\mathbf{A}+\exp (\alpha t) \mathbf{B}+\exp (\beta t) \mathbf{C}] \mathbf{N}(0)
$$

From equation 9, we obtain the survivor functions $G_{1}(t)=\frac{N_{1}(t)}{N(0)}$ and $G_{2}(t)=\frac{N_{2}(t)}{N(0)}$ as

$$
\begin{aligned}
G_{1}(t) & =\frac{\lambda_{1} \mu_{2}}{\alpha \beta}+\frac{\lambda_{1}\left(\alpha+\mu_{2}\right)}{\alpha(\alpha-\beta)} \exp (\alpha t) \\
& +\frac{\lambda_{1}\left(\beta+\mu_{2}\right)}{\beta(\beta-\alpha)} \exp (\beta t), \\
G_{2}(t) & =\frac{\lambda_{2} \mu_{1}}{\alpha \beta}+\frac{\mu_{2}\left(\alpha+\mu_{1}\right)}{\alpha(\alpha-\beta)} \exp (\alpha t) \\
& +\frac{\mu_{2}\left(\beta+\mu_{1}\right)}{\beta(\beta-\alpha)} \exp (\beta t),
\end{aligned}
$$

respectively. Based on these survivor functions for each level, we have

$$
\lim _{t \rightarrow \infty} G(t)=\frac{\lambda_{1} \mu_{2}+\lambda_{2} \mu_{1}}{\alpha \beta}
$$

The choice of a course by a candidate may be guided by the magnitude of $G_{1}(t)$ or $G_{2}(t)$ depending on whether the candidate is applying for admission into 100 Level or 200 Level, respectively. A UTME candidate, for example, who is indecisive on two preferred courses, say $C 1$ and $C 2$, may select $C 2$ if, on the average, the survivor rate for the course $C 2$ is higher than that of course $C 1$. 
Ekhosuehi V.U. and Oyegue, F.O. African Journal of Applied Statictics, Vol. 3 (1), 2016, 59 - 67. Survivor rates of new entrants into an undergraduate degree programme under clearance uncertainty.

\section{Illustrative example}

To get an insight into the behaviour of the survivor functions $G_{1}(t)$ and $G_{2}(t)$, the simulation of observations on the number of candidates cleared and the number of candidates who leave the system in the year of entry is a helpful tool. We randomly generate ten replicates for each set of observations using the function rand in the MATLAB environment. The MATLAB command "rand" generates uniform random numbers. This command in MATLAB returns a list of simulated sequence of observations which are used to compute the propensities, $\lambda_{i}$ and $\mu_{i}$. In calculating $\lambda_{i}$ and $\mu_{i}$, we set the number of candidates offered provisional admission to be 100 and 20 for the 100 Level and the 200 Level, respectively. We assume that the number of candidates cleared is partly constant and then access the simulated sequence of observations and the propensities with

$\mathrm{clc}$

$\mathrm{n}=10 ; \operatorname{ad}=[70+\operatorname{rand}(1, \mathrm{n}) * 30] ;$

$\mathrm{rj}=100-\mathrm{ad} ; \mathrm{v} 1=\mathrm{mean}(\mathrm{ad}) / 100$,

$\mathrm{u} 1=\operatorname{mean}(\mathrm{rj}) / 100$,

$\operatorname{ad} 2=[10+\operatorname{rand}(1, \mathrm{n}) * 10] ;$

r j $2=20-\operatorname{ad} 2 ; \quad v 2=$ mean $(\operatorname{ad} 2) / 20$,

$\mathrm{u} 2=\operatorname{mean}(\mathrm{rj} 2) / 20$,

However, one should not attach undue importance to this simulation as the purpose of this illustration is to give an insight on the behaviour of the survivor functions. From the simulated observations, we obtain the plot in Figure 2, which displays the survivor rates for the 100 Level and the 200 Level. As shown, it is easy to see that the sum of $G_{1}(t)$ and $G_{2}(t)$ lies within the bounds 0 and 1 , and that the survivor rates are increasing monotonically with time (in days). This is what is expected as in the real life situation the clearance of candidates into any course does not reduce the number already cleared but gradually adds to it until the desired capacity of the course is attained. This monotone pattern may be attributed to the time spent on verification of documents, the time required to certify and collate all relevant documents for the clearance, the traffic encountered in the payment of acceptance fees, etc. so much so that the clearance exercise is not instantaneous.

\section{Concluding remarks}

This paper characterises in a theoretical way the survivor rates of candidate who has been offered (provisional) admission into a course of study when they undergo the clearance exercise. It turns out that the survivor function derived in this study, which utilises a network model with constant risk as its theoretical underpinning, is a monotonically increasing function. The survivor function may be used as a decision making tool on the choice of courses. Nonetheless, this approach is not meant to replace the candidate's intuition, but to guide the candidate on decisions based on the survivor rates. The results obtained from the simulation suggest that the survivor function is a descriptive realisation of the clearance process. However, since it has not been validated with real life data, it is still only a possible explanation. To validate the survivor function, it would be necessary to implement policy changes de- 
Ekhosuehi V.U. and Oyegue, F.O. African Journal of Applied Statictics, Vol. 3 (1), 2016, 59 - 67.

Survivor rates of new entrants into an undergraduate degree programme under clearance uncertainty.

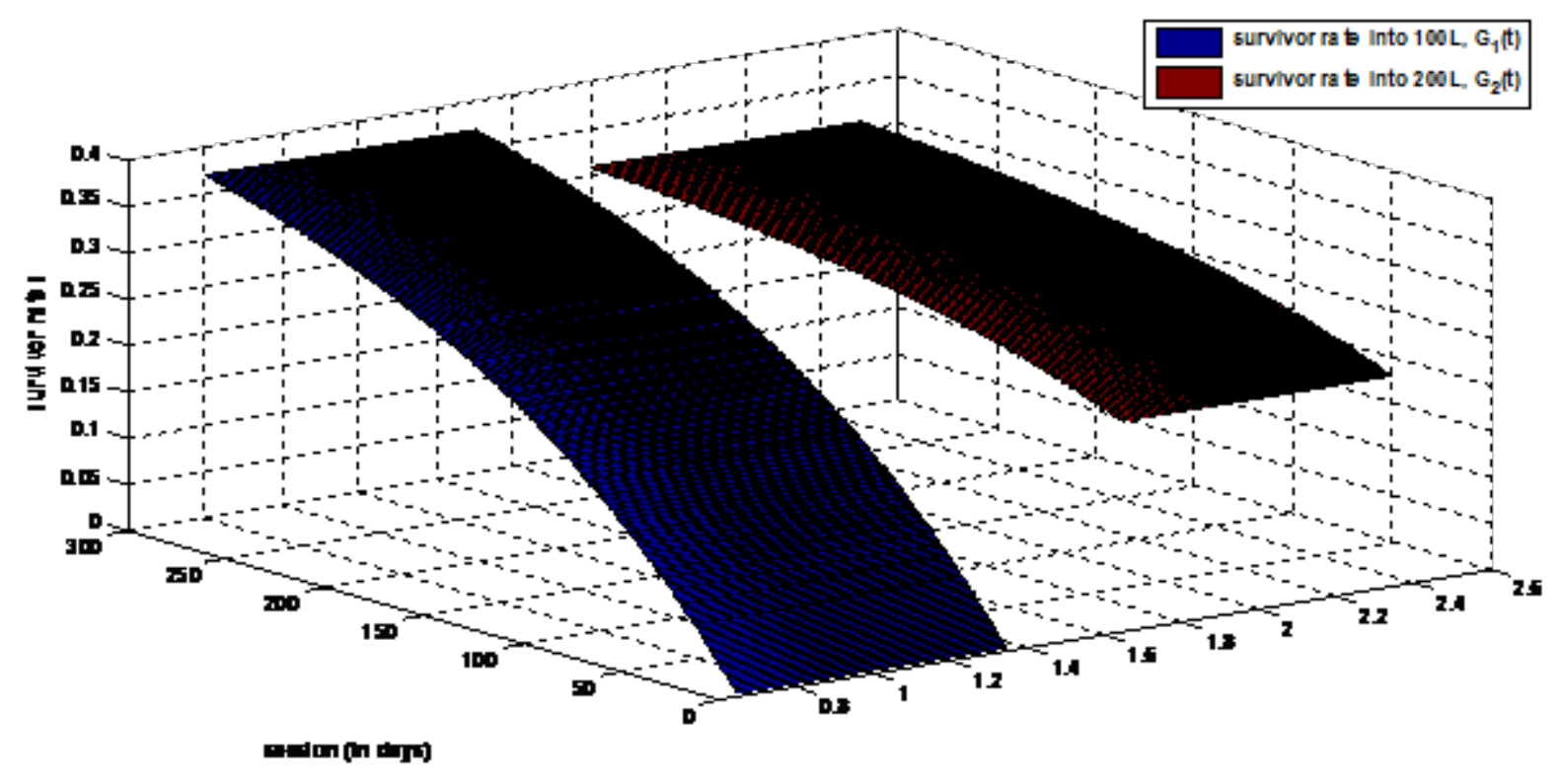

Fig. 2. Survivor rates for the simulated observations.

signed to affect the propensities $\lambda_{i}$ or $\mu_{i}$ and demonstrate that the monotonically increasing pattern do occur. This is not yet done.

\section{References}

Ekhosuehi, V. U., 2016. On the evolution of academic staff structure in a university setting. To appear in: Journal of Mathematics and Applications, vol. 39.

Ekhosuehi, V. U. and Osagiede, A. A., 2013. A proposed Markov model for predicting the structure of a multi-echelon educational system in Nigeria. Monografias Matematicas Garcia de Galdeano, vol. 38, pp. p65-74.

Gani, J., 1963. Formulae for projecting enrolments and degrees awarded in universities. Journal of the Royal Statistical Society Series A, vol. 126, (3), pp. p400-409

Johnes, J., 2015. Operational research in education. European Journal of Operational Research, vol. 243, (3), pp. p683-696. doi: 10.1016/j.ejor.2014.10.043

Jimenez, J. D. and Salas-Velasco M., 2000. Modeling educational choices. A binomial logit model applied to the demand for higher education. Higher Education, vol. 40(3), pp. p293-311.

Mancebon, M. J. and Muniz, M. A., 2008. Private versus public high schools in Spain: disentangling managerial and programme efficiencies. Journal of the Operational Research Society, vol. 59 (7), pp. p892-901.

Nicholls, M. G., 2009. The use of Markov models as an aid to the evaluation, planning and benchmarking of doctoral programs. Journal of the Operational Research Society, vol. 60(9), pp. p1183-1190. 